\title{
VEGF-A not Ang2 mediates endothelial-like differentiation of immature DCs by ERK1/2 signaling in the microenvironment of human colon adenocarcinoma
}

\author{
JING LU*, KANGDONG LIU*, JIMIN ZHAO, JUN ZHAO, JUNFEN MA, \\ HONGYAN YANG, YOUTIAN HUANG, ZHENZHU QIN, RUIHUA BAI, LILI JIANG, \\ FENGSHOU LV, PEI LI, WENHAI YAN, MINGYAO ZHAO and ZIMING DONG
}

Department of Pathophysiology, School of Basic Medical Sciences, Zhengzhou University, Zhengzhou 450001, P.R. China

Received December 30, 2010; Accepted February 24, 2011

DOI: 10.3892/ijo.2011.989

\begin{abstract}
Endothelial-like differentiation of dendritic cells (DCs) is an interesting and significant phenomenon, which is worth further investigation. Here, we show that the tumor microenvironment derived from the supernatant of the SW620 human colon adenocarcinoma cell line and colon adenocarcinoma tissue homogenate can promote immature DCs (iDCs) to differentiate from the DC pathway toward endothelial cells, while the peri-carcinoma homogenate supernatant does not have this role. Inhibition of angiopoietin-2 (Ang2) in the supernatant by its antibody has no obvious influence on the endotheliallike differentiation. In contrast, inhibition of vascular endothelial growth factor-A (VEGF-A) blocked the differentiation. During the course of differentiation, a sustained activation of ERK1/2 was detected. PD 98059 blocked the phosphorylation of ERK1/2 as well as the endothelial-like differentiation of iDCs. Inhibition of VEGF-A also blocked the phosphorylation of ERK1/2. These data suggest that VEGF-A not Ang2 mediates endothelial-like differentiation of iDCs by ERK1/2 signaling in the microenvironment of human colon adenocarcinoma.
\end{abstract}

\section{Introduction}

Dendritic cells (DCs) are antigen-presenting cells that play a key role in the initiation and regulation of immune responses. However, recent research shows that a novel leukocyte subset within murine and human ovarian carcinoma coexpressed

Correspondence to: Dr Ziming Dong, Department of Pathophysiology, School of Basic Medical Sciences, Zhengzhou University, Zhengzhou 450001, P.R. China

E-mail: dongzm@zzu.edu.cn

${ }^{*}$ Contributed equally

Key words: dendritic cells, colon adenocarcinoma, endothelial-like differentiation, angiopoietin-2, vascular endothelial growth factor, extracellular signal regulated kinase endothelial cell (EC) and DC markers, and has proved that murine bone marrow-derived DCs could undergo endothelialization in vitro after incubation at a high level of vascular endothelial growth factor-A (VEGF-A) (1-4). CD34+ progenitor cells, which are capable of developing into DCs, with the addition of conditioned medium of murine Lewis lung carcinoma cells redirected their differentiation into ECs (5). Tumor-associated DCs (TADCs) incubated additionally with VEGF and oncostatin M could trans-differentiate into endothelial-like cells (ELCs), which might be an alternative pathway of tumor angiogenesis (6). These reports show that DC progenitor or DCs can trans-differentiate into ELCs, possibly contributing to vasculogenesis in the adult. Thus, DCs might exert an important impact on the neovascularization process in different physiopathological conditions (7).

We previously reported that the tumor microenvironment derived from the supernatant of human esophageal squamous cell carcinoma cell line EC9706 could induce immature DCs (iDCs) to differentiate into ELCs. Inhibition of VEGF-A in the supernatant blocked the endothelial-like differentiation (8). We questioned 1) is the contribution of ELCs to vasculogenesis organ-dependent? 2) is there some better way to simulate a tumor microenvironment in vitro than by using tumor cell culture supernatant or by adding some cytokine (5-9)? 3) can the normal tissue microenvironment promote the endothelial-like differentiation of iDCs? 4) besides VEGF-A, are other pro-angiogenic factors involved in this differentiation?

Colorectal cancer is the third most common form of human cancer, and it is the second most common cause of cancerrelated death in the Western world (10). In this study, we examined the possibility of the endothelial-like differentiation of iDCs in the microenvironment derived from the supernatant of human colon adenocarcinoma cell line SW620 and colon adenocarcinoma tissue homogenate, and investigated the role of angiopoietin-2 (Ang2), VEGF-A, and ERK1/2 signaling in endothelial-like differentiation of iDCs. Results show that both the supernatant of SW620 cell culture and colon adenocarcinoma tissue homogenate can induce iDCs to differentiate from the DC pathway toward an EC pathway, but the pericarcinoma tissue homogenate supernatant does not have this 
role. In addition, VEGF-A not Ang2 in the supernatant mediates endothelial-like differentiation of iDCs by ERK1/2 signaling.

\section{Materials and methods}

Culture of primary human umbilical vein endothelial cells. The aseptic cords were contributed by healthy parturient donors from the Third Affiliated Hospital, Zhengzhou University School of Medicine. The method of isolation, culture and identify of primary human umbilical vein endothelial cells (HUVECs) is as previously described (8).

Preparation of SW620 cell culture supernatant and homogenate supernatant of colon adenocarcinoma and peri-carcinoma. SW620 cells were cultured in RPMI-1640 medium with $10 \%$ fetal calf serum. The cells were replenished with fresh medium after reaching 60-80\% confluence. The supernatant was collected and filtered after 24-h incubation (5). Fresh tumor specimens of poorly differentiated colon adenocarcinoma tissue and peri-carcinoma tissue $(>5 \mathrm{~cm}) 6$ cases (without preoperative radiotherapy and chemotherapy) were collected to prepare to the homogenate supernatant by grinding and centrifuge. The above specimens were contributed by donors from the Henan Tumor Hospital.

Induction of endothelial-like cells from iDCs. Peripheral blood mononuclear cells (PBMCs) were harvested from healthy adult volunteers by density gradient centrifugation over Ficoll (6) and seeded in 24 -well plates at $2 \times 10^{9} / 1$ for $3 \mathrm{~h}$. The adherent cells (monocytes) were induced toward DCs with rhGM-CSF $100 \mu \mathrm{g} / 1$ (Amoytop), rhIL-4, $5 \mu \mathrm{g} / 1$ (PeproTech). SW620 supernatant (40\%) was added at the end of day 2. After 7 days of induction with SW620 supernatant, induced cells were harvested on day 9 (i.e. 2+7) for study. Parallel culturing of control DCs also were harvested on day 9. To detect the effect of Ang2 and VEGF-A on the differentiation of iDCs, Ang2 antibody ( $1 \mu \mathrm{g} / \mathrm{ml})$ or VEGF-A antibody $(1 \mu \mathrm{g} /$ $\mathrm{ml}$ ) and isotype control $\mathrm{IgG}$ (Santa Cruz) were first mixed with the SW620 supernatant.

Immunofluorescence analysis. Induced cells, control DCs and the HUVECs were seeded in 96-well plates and incubated for $24 \mathrm{~h}$, then fixed with $4 \%$ paraformaldehyde for $30 \mathrm{~min}$. Rabbit anti-human vWF (1:200; Santa Cruz) as the primary antibody and FITC-conjugated goat anti-rabbit IgG as the secondary antibody were used. For detection of nuclear translocation of phospho-p44/42 MAPK/ERK Ab (Thr202/Tyr204; 1:200; Cell Signaling) as the primary antibody and FITC-conjugated goat anti-rabbit IgG as the secondary antibody were used, and the nuclear stain PI (Sigma) $200 \mu \mathrm{g} / \mathrm{ml}$ was used. The procedure was followed by the immunocytochemistry. Cells with green fluorescent particles in the cytoplasm were counted as having positive expression.

Flow cytometry (FCM) analysis of DC surface markers. Cells of the SW620 supernatant group, and its control DCs were harvested on day 9. The cells were resuspended in PBS with concentration $1 \times 10^{6} / \mathrm{ml}$. Monoclonal antibodies against human CD86, CD1a (FITC, BD), CD11c (PE, BD) $\left(20 \mu 1 / 10^{6}\right.$ cells $)$ were incubated with the cells for $30 \mathrm{~min}$ in dark at $4^{\circ} \mathrm{C}$. The cells were washed with PBS, resuspended in PBS and analyzed by flow cytometry.

Western blot analysis. Total protein concentration of cells in each group was measured by the Bradford method. Cell lysates $(50 \mu \mathrm{g})$ were resolved to $10 \%$ SDS-PAGE gel and transferred to PVDF membrane. vWF Ab (1:300), CD144 Ab (1:1000; Cell Signaling), phospho-p44/42 MAPK/ERK Ab (Thr202/ Tyr204; 1:1000; Cell Signaling), anti-p44/42 MAPK/ERK Ab (Thr202/Tyr204; 1:1000; Cell Signaling) were added, respectively overnight at $4^{\circ} \mathrm{C}$. HRP-IgG secondary antibody was incubated for $2 \mathrm{~h}$ at room temperature. The membranes were visualized with ECL. The Gel Doc Imaging System was used for detecting the gray value of the protein bands.

In vitro angiogenesis assay. The cells of SW620 supernatant group, control DCs and HUVECs were digested and washed twice and resuspended with PBS. The cells were seeded in the 96-well plates (coated with fibronectin for $45 \mathrm{~min}$ ) with $1 \times 10^{5}$ cells/well, in quadruplicate, and were cultured with ECM at $37^{\circ} \mathrm{C}$ with $5 \% \mathrm{CO}_{2}$. The cells were observed with an inverted microscope.

DiI-Ac-LDL and India ink uptake assay $(6,11)$. The induced cells and control were seeded in 96-well plates and incubated for $36 \mathrm{~h}$, then, $10 \mu \mathrm{g} / \mathrm{ml}$ Dil-Ac-LDL (Biomedical Technologies Inc.) was added and the cells were further incubated at $37^{\circ} \mathrm{C}$ for $4 \mathrm{~h}$. The medium containing DiI-Ac-LDL was removed, and washed 3 times with PBS, then $10 \mu \mathrm{l} / \mathrm{ml}$ India ink was added into the medium and the cells were incubated at $37^{\circ} \mathrm{C}$ for another $4 \mathrm{~h}$. The India ink medium was removed and the cells were washed 3 times with PBS. Cells were observed with an inverted fluorescence microscope.

Detection of Weibel-Palade bodies by transmission electron microscopy. The cells of the SW620 supernatant group and the control DCs were digested by $0.25 \%$ trypsin and centrifuged at $2000 \mathrm{r} / \mathrm{min}$ for $10 \mathrm{~min}$, and pro-fixed with $2.5 \%$ glutaraldehyde overnight. Then, the cells were post-fixed by $1 \%$ osmic acid, dehydrated with alcohol gradient, dehydrated with $100 \%$ acetone, embedded in epoxy resins 812,815 , sliced by Leica ultramicrotomy, and double electron stained with uranyl acetate and citric acid. The photographs were taken by transmission electron microscopy (TEM) (Hitachi H-7500).

Statistical analysis. Data were expressed as means \pm standard deviation (SD) with at least three separate experiments and analyzed by One-way ANOVA and q test. Significance was defined as $\mathrm{p}<0.05$.

\section{Results}

Endothelial-like differentiation of iDCs induced by SW620 supernatant. The iDCs were induced by SW620 supernatant for 7 days. The induced cells were slender and tended to form tube-like structures, and some cells were arrayed into cordlike structures (Fig. 1A), a typical appearance of ECs. The control DCs were round (Fig. 1A). To identify the effect of SW620 supernatant on the expression of DC surface markers 


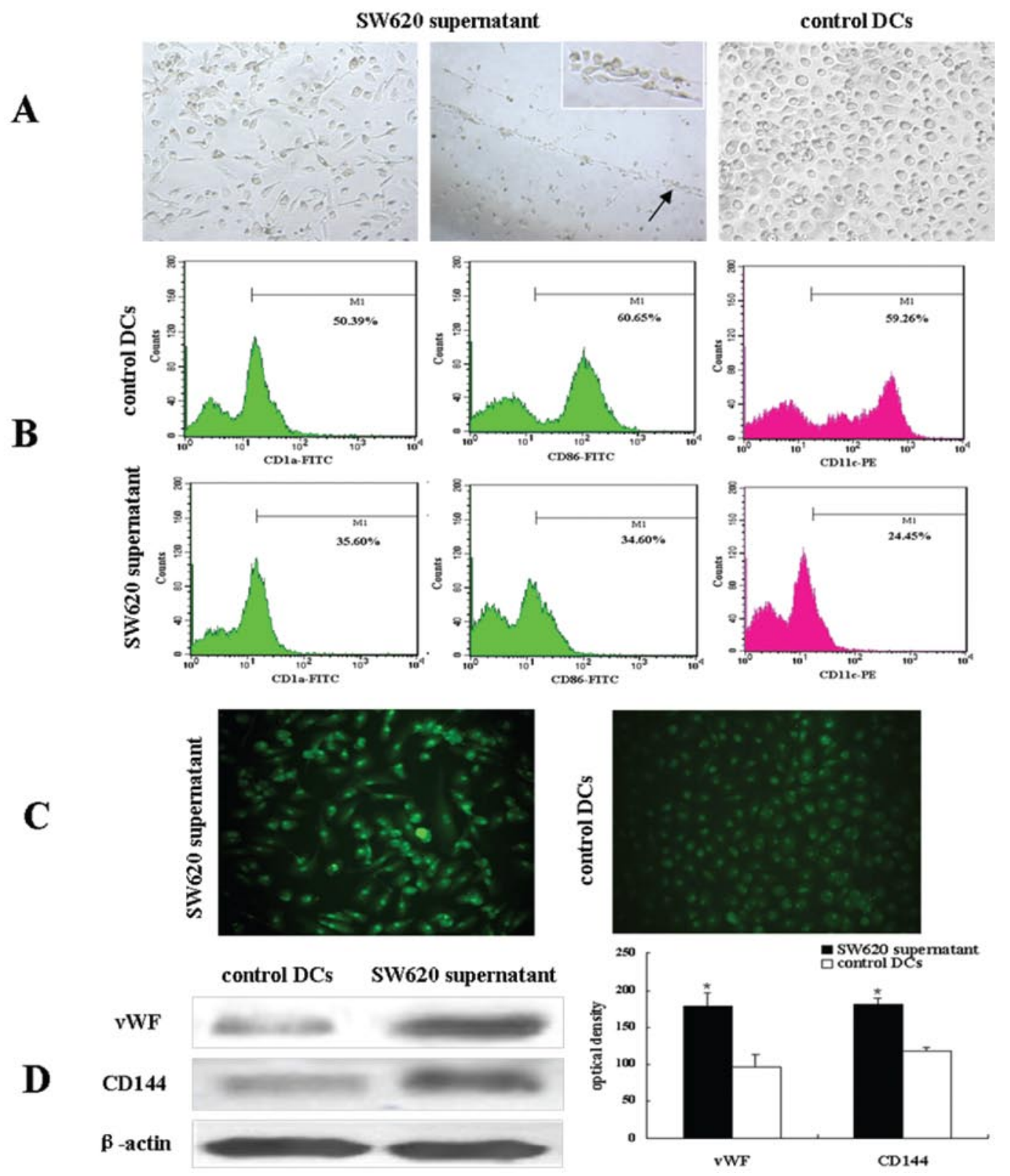

Figure 1. Cell morphology and associated antigen expression of the endothelial-like differentiation of iDCs. (A) Morphology change of the induced cells. PBMCs were incubated in rhGM-CSF/rhIL-4, and the 40\% SW620 supernatant was added at the end of day 2. The cells of the SW620 supernatant group presented a tendency toward tube-like structures and strip-shaped structures after adding SW620 supernatant for 7 days. The control DCs were parallel cultured with rhGM-CSF/rhIL-4 on day 9. Magnification x200. (B) FCM analysis of the expression rate of DC specific markers in induced cells and control DCs. (C) The expression of the EC marker vWF in induced cells by immunofluorescence. vWF expression in the cytoplasm of SW620 supernatant group and control DCs. Magnification x200. (D) Western blot detection of the CD144 and vWF in the induced cells. Results shown are the mean \pm SD (n=3). " $\mathrm{p}<0.01 \mathrm{vs}$. control DCs.

in induced cells, CD86, CD1a and CD11c were detected by FCM. The expression levels of CD86, CD1a and CD11c were decreased in induced cells compared with the control DCs (Fig. 1B).

Next, we identified the effect of SW620 supernatant on the expression of EC surface markers in induced cells by Western blot and immunofluorescence analysis. The expression levels of vWF and CD144 were increased in induced cells (178.4 \pm 18.45 , 180.2 \pm 9.09$)$ compared with the control DCs $(95.4 \pm 16.80$, 118.0. \pm 4.74$)(n=3, p<0.01)$ (Fig. 1D). Immunofluorescence analysis also revealed the enhanced expression of vWF in induced cells (Fig. 1C).
To demonstrate that the cells induced by SW620 supernatant had the endothelial ability to form into tube-like structures, the induced cells, control DCs and HUVECs were seeded onto fibronectin-coated plates. The results showed that the induced cells appeared in fusiform shape and tendency for vortex distribution (Fig. 2Aa, b), which was very similar to HUVECs (Fig. 2Ad). They tended to form a tube-like structure (Fig. 2Ac), which was similar to the tube-like structure formed by HUVECs (Fig. 2Ae). In contrast, the cells were distributed evenly in control DCs (Fig. 2Af).

Although shared by other cells as macrophages and monocytes, uptake of DiI-Ac-LDL is considered to be one of the 


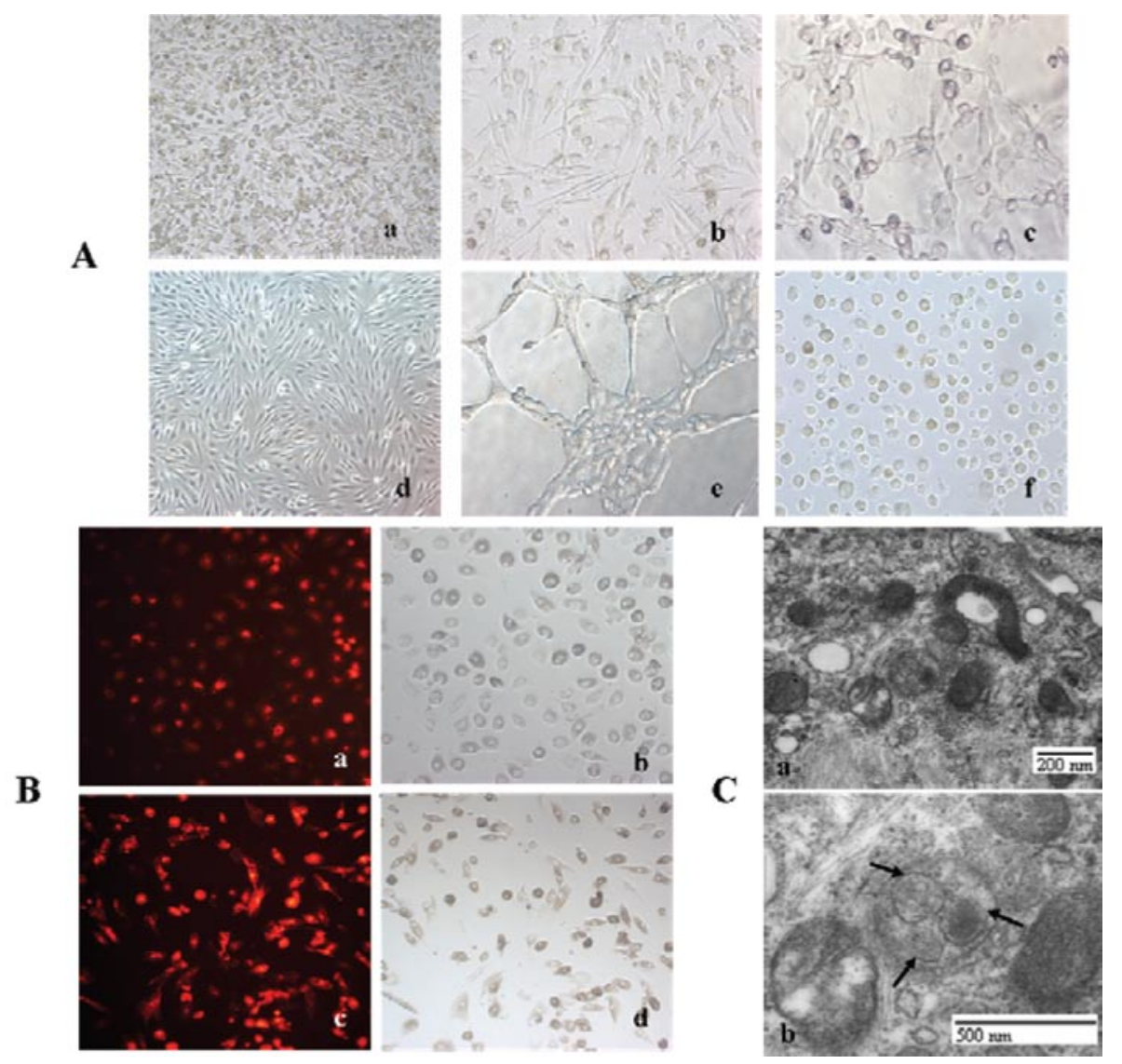

Figure 2. Analysis of the functional change of the endothelial-like differentiation of iDCs. (A) The formation of tube-like structures on fibronectin-coated plates. The cells of the SW620 supernatant group appeared with fusiform shapes and a vortex distribution (a and b). The cells of iDCs induced group had a tendency to form tube-like structures (c). The HUVECs appeared with fusiform shapes, a vortex distribution (d) and tube-like structures (e). The control DCs (f) appeared round and evenly distributed. Magnifications for (a) and (d) are x100. Magnifications for (b), (c), (e) and (f) are x200. (B) DiI-Ac-LDL and India ink uptake assay of control DCs (a) and the SW620 supernatant group (c). (b) and (d) are phase contrast photographs that are related to (a) and (c), respectively, which show no india ink uptake. Magnification x200. (C) Acquisition of the endothelial feature, WP body, as seen by TEM. (a) Organized structure of the cells of SW620 supernatant group. Magnification x20,000. (b) The presence of WP bodies in the cells of SW620 supernatant group. Arrow shows WP body. Magnification x50,000.

typical functions of ECs $(12,13)$. However, macrophages and monocytes not only show uptake of DiI-Ac-LDL but also take up India ink (11). In our previous research we also proved that PBMCs had weak uptake of DiI-Ac-LDL, and strong uptake of India ink (8). The induced cells did not take up India ink (Fig. 2Bd), which excluded the possibility that monocytes and macrophages were mixed in with the induced cells. The increased uptake of DiI-Ac-LDL in induced cells showed that the iDCs tended to differentiate toward ECs (Fig. 2Bc). In addition, EC-specific functional structure of WP body was detected by TEM. The results showed that WP bodies and plenty of mitochondria were present in induced cells (Fig. 2C). No WP body was found in control DCs.

Thus, the above results showed that iDCs differentiated into ELCs in the colon carcinoma microenvironment derived from SW620 supernatant, both phenotypically and functionally.

The effect of homogenate supernatant of colon adenocarcinoma and peri-carcinoma tissue on endothelial-like differentiation of iDCs. The iDCs were induced by homogenate supernatant of colon adenocarcinoma and peri-carcinoma for 7 days. The cells induced by carcinoma homogenate supernatant were slender, while the cells induced by peri-carcinoma homogenate supernatant were round, similar to the appearance of control DCs (Fig. 3A).

The expression levels of vWF and CD144 were increased in cells induced by carcinoma homogenate supernatant $(205.3 \pm 2.52,213.7 \pm 5.13)$ compared with the peri-carcinoma group $(156.0 \pm 4.00,148.3 \pm 2.52)(\mathrm{n}=3, \mathrm{p}<0.01)$ and control DCs $(147.0 \pm 5.57,140.7 \pm 2.52)(n=3, p<0.01)$. There is no obvious difference between the peri-carcinoma group and control DCs ( $n=3, p>0.05)$ (Fig. 3D). Immunofluorescence analysis also revealed the enhanced expression of vWF in induced cells (Fig. 3B). The induced cells did not take up India ink (Fig. 3C lower). The increased uptake of DiI-Ac-LDL in carcinoma homogenate supernatant group compared with uptake in the peri-carcinoma group and in control DCs. There is no obvious difference between the peri-carcinoma group and control DCs (Fig. 3C upper).

The blocking of Ang2 in SW620 supernatant can not inhibit the endothelial-like differentiation of iDCs. To determine the role of Ang2 in the endothelial-like differentiation of iDCs induced by SW620 supernatant, Ang2 $\mathrm{Ab}(1 \mu \mathrm{g} / \mathrm{ml})$ was used to investigate its effect on cell morphology, the expression of vWF and CD144, and the function of DiI-Ac-LDL uptake. 


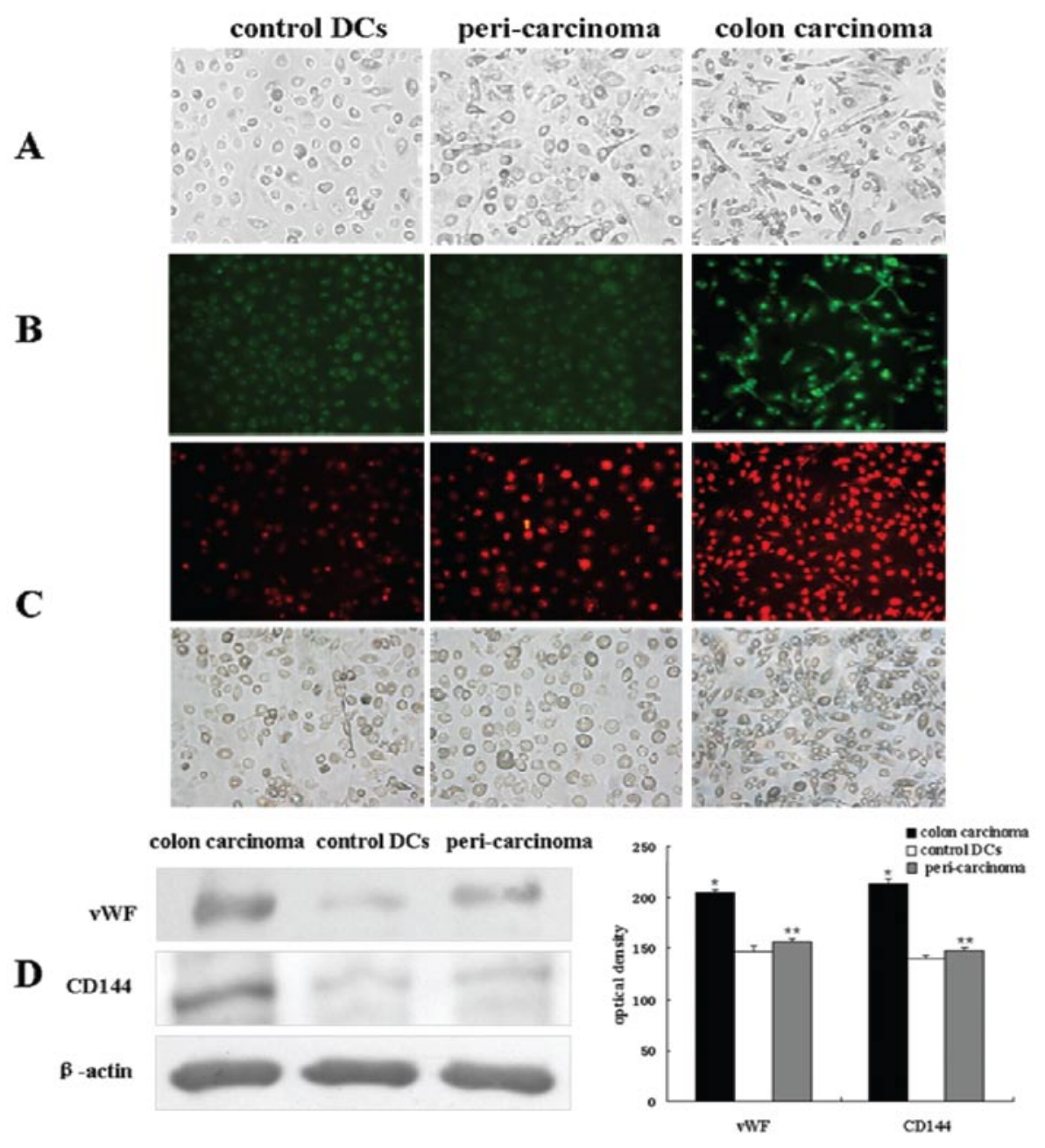

Figure 3. Analysis of the effect of colon adenocarcinoma and peri-carcinoma tissue homogenate supernatant on the endothelial-like differentiation of iDCs. (A) Morphology change of the induced cells. The cells of the colon carcinoma group appeared with fusiform shapes after adding colon adenocarcinoma tissue homogenate supernatant for 7 days. The cells of the peri-carcinoma group had no obvious morphology change, similar to control DCs. Magnification x200. (B) The expression of vWF in the induced cells by immunofluorescence. Magnification x200. (C) DiI-Ac-LDL and India ink uptake assay. Upper pictures show DiI-Ac-LDL uptake, lower pictures show no india ink uptake respectively. Magnification x200. (D) Western blot detection of the vWF and CD144 in the induced cells. Results shown are the mean $\pm \mathrm{SD}(\mathrm{n}=3) .{ }^{*} \mathrm{p}<0.01$ vs.control DCs and peri-carcinoma group; ${ }^{* *} \mathrm{p}>0.05$ vs. control DCs.

The induced cells showed slender spindles. However, there was no difference between the blocking Ang2 group and the induced cells (Fig. 4A). The expression levels of vWF and CD144 were not different among the blocking Ang2 group $(214.3 \pm 5.69,191.0 \pm 3.61)$, the SW620 supernatant group $(218.7 \pm 1.53,197.0 \pm 2.00)$, and isotype control $(219.0 \pm 3.61$, 191.7 \pm 2.52$)(\mathrm{n}=3, \mathrm{p}>0.05)$ (Fig. 4D). Immunofluorescence showed that the expression level of vWF of the blocking Ang2 group was similar to the induced cells (Fig. 4B). The uptake of DiI-Ac-LDL showed no difference between the blocking Ang2 group and the induced cells (Fig. 4C).

The blocking of VEGF-A in SW620 supernatant results in the inhibition of the endothelial-like differentiation of iDCs. To determine the role of VEGF-A in the endothelial-like differentiation of iDCs, VEGF-A Ab $(1 \mu \mathrm{g} / \mathrm{ml})$ was used to investigate its effect. The induced cells showed slender spindles. There was almost no slender branching in cells of the blocking VEGF-A group, which was similar to control DCs (Fig. 5A). Immunofluorescence showed that vWF expression decreased in the cells of the blocking VEGF-A group compared with the induced cells (Fig. 5B). Uptake assay showed that the uptake of DiI-Ac-LDL decreased in the cells of the VEGF-A blocking group. There was no difference between the blocking VEGF-A group and control DCs (Fig. 5C).

ERK1/2 signaling is involved in endothelial-like differentiation of iDCs in the SW620 supernatant microenvironment. To determine the function of ERK1/2 signaling in the process of endothelial-like differentiation of iDCs, we examined if ERK1/2 signaling was activated by the SW620 supernatant. Results showed that the phosphorylation of ERK1/2 increased in a time-dependent manner in the differentiating iDCs. SW620 supernatant stimulates a strong phosphorylation of $\mathrm{p} 44 / 42$ ERK1/2 after 15 min of incubation. PD98059 is a selective inhibitor of MEK1/2, the upstream regulator of phosphorylation of ERK1/2. Therefore, we used PD98059 $50 \mu \mathrm{M}$ to block MEK1/2 to determine the phosphorylation of ERK1/2. Results showed that phosphorylation of ERK1/2 was obviously inhibited in the presence of PD98059 (n=3, p<0.05) (Fig. 6A). 


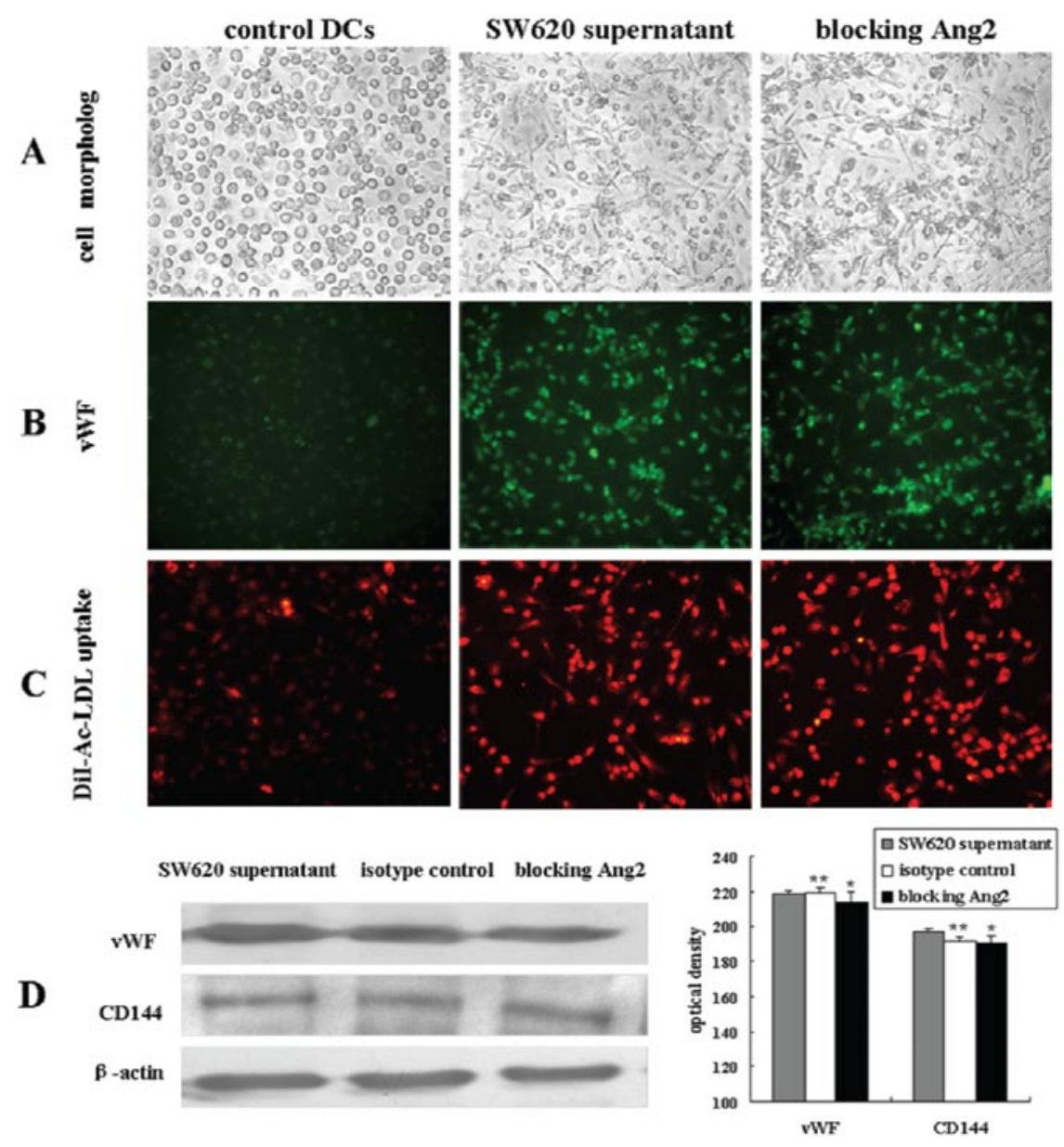

Figure 4. Blocking Ang2 in SW620 supernatant had no effect on the endothelial-like differentiation of iDCs. (A) No morphological changes after blocking Ang2 in SW620 cell supernatant by Ang2 Ab (magnification x200). The expression of vWF (B) and DiI-Ac-LDL uptake (C) after blocking Ang2 (magnification x200). (D) Western blot analysis of the expression of vWF and CD144 after blocking Ang2. Results shown are the mean \pm SD ( $n=3$ ). " $p>0.05$ vs. SW620 supernatant group and isotype control; ${ }^{* *}$ p $>0.05$ vs. SW620 supernatant group.

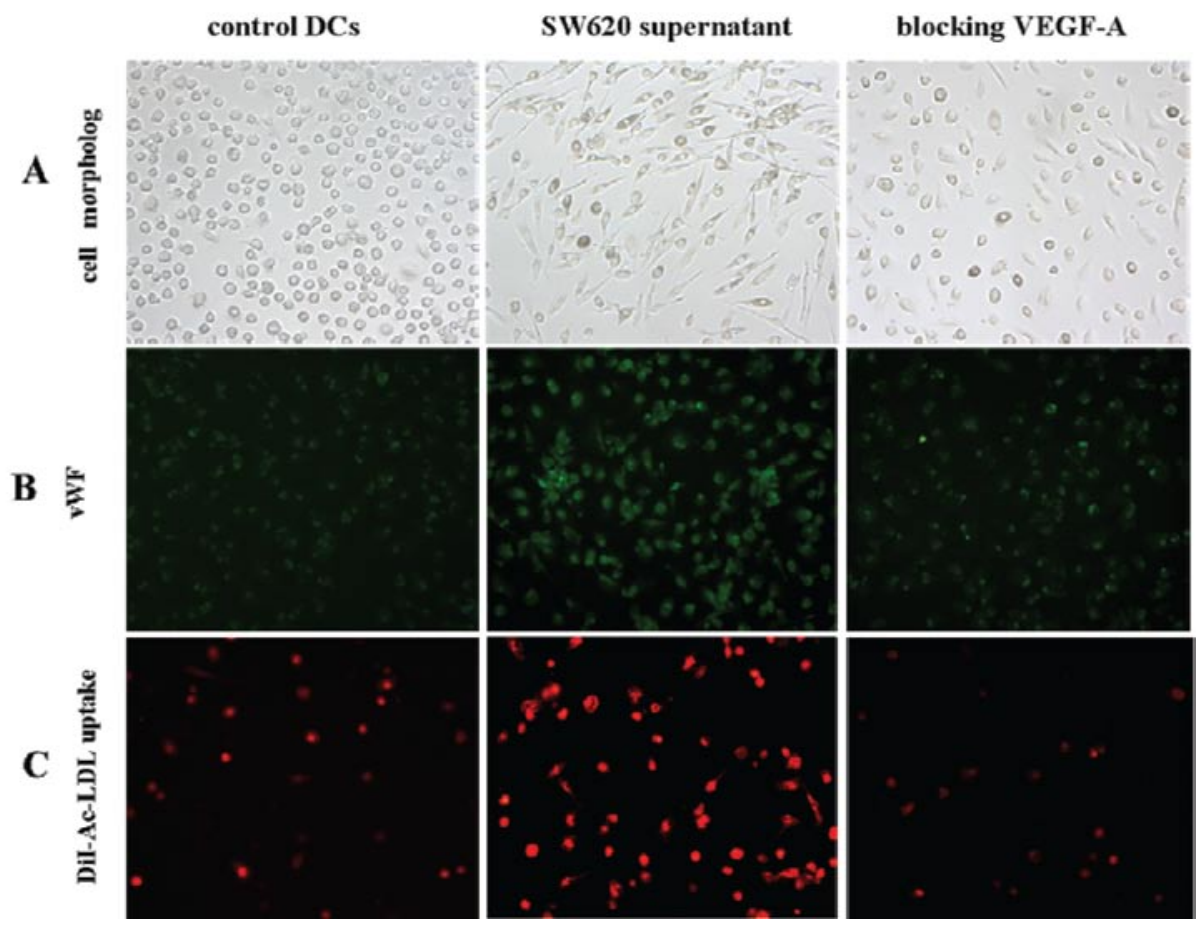

Figure 5. Blocking VEGF-A in SW620 supernatant resulted in the inhibition of the endothelial-like differentiation of iDCs. (A) Morphological changes after blocking VEGF-A in SW620 supernatant by VEGF-A Ab (magnification x200). (B) Immunofluorescence analysis of the decreasing expression of vWF after blocking VEGF-A in SW620 supernatant (magnification x200). (C) Blocking VEGF-A decreasing the DiI-Ac-LDL uptake (magnification x200). 


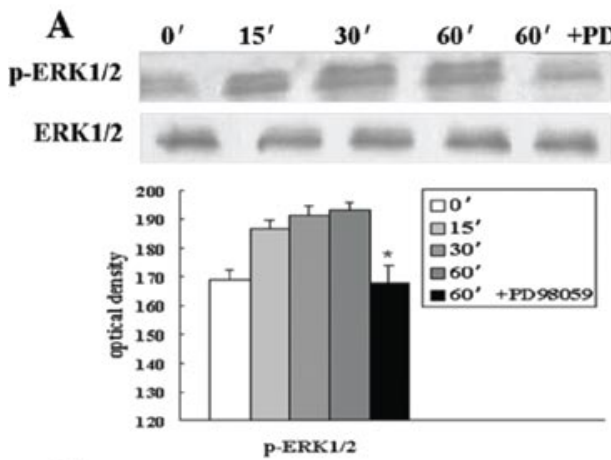

B SW620 supernatant SW620 supernatant + PD
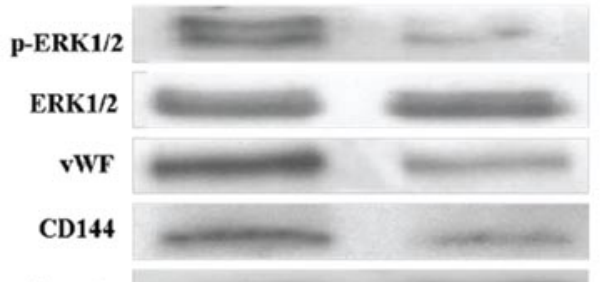

$\beta$-actin

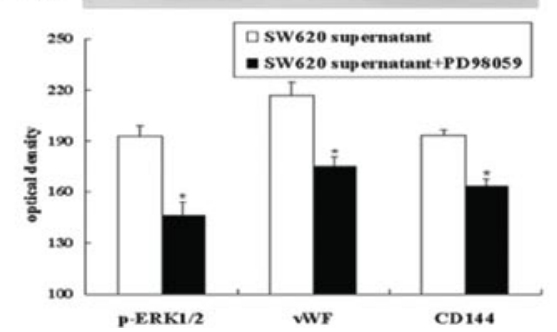

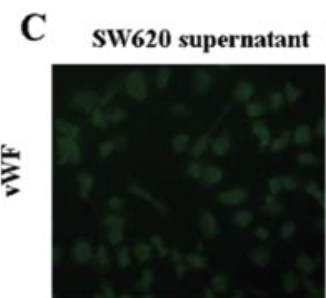
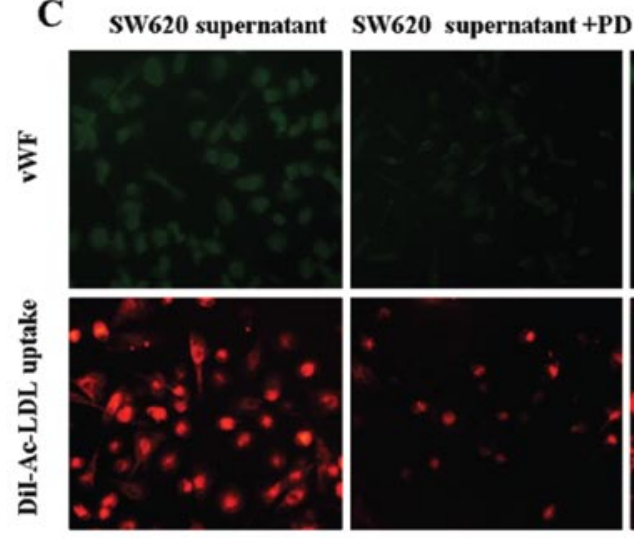

D

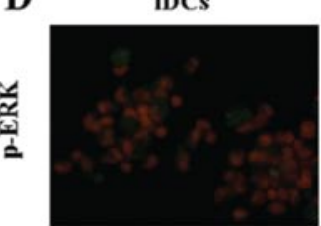

SW620 supernatant
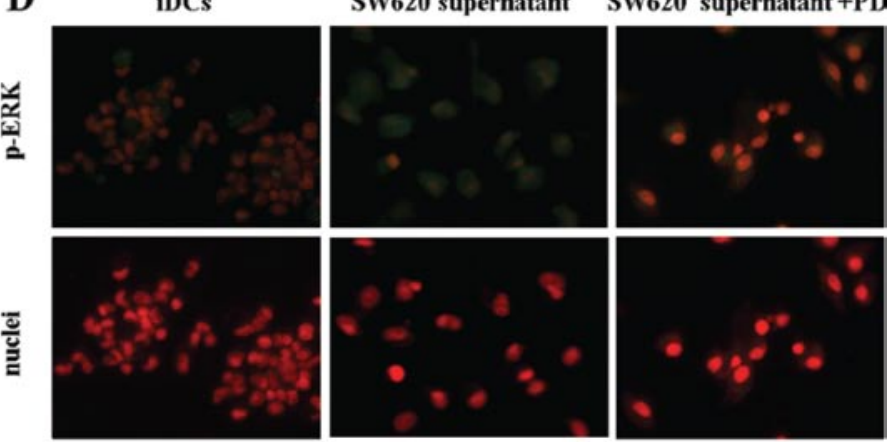

Figure 6. PD98059 blocking the activation of ERK1/2 and inhibiting endothelial-like differentiation of iDCs. (A) ERK1/2 was activated after 15 min of incubation with SW620 supernatant and sustained throughout the period of $60 \mathrm{~min}$ in iDCs, and the activation of ERK1/2 was inhibited by PD98059. Results shown are means \pm SD (n=3). " p $<0.05$ vs. 30', 60' group. PD: PD98059 $50 \mu \mathrm{M}$. (B) Ten $\mu$ M PD98059 blocked the activation of ERK1/2 and decreased the expression of vWF and CD144 in the cells of SW620 supernatant group. Results shown are the mean \pm SD (n=3). ${ }^{*}$ p $<0.01$ vs. SW620 supernatant group. (C) Ten $\mu \mathrm{M}$ PD98059 decreased the expression of vWF and the DiI-Ac-LDL uptake in cells of SW620 supernatant + PD as seen by immunofluorescence (magnification $x 200$ ). (D) PD98059 inhibited nucleus translocation of phospho-ERK1/2 in iDCs treated with SW620 supernatant for 7 days. iDCs incubated with SW620 supernatant showed the presence of phospho-ERK1/2 (green) in the nuclei (red) of endothelial-like differentiation of iDCs, and phospho-ERK1/2 as well as nuclear translocation were inhibited in the presence of PD98059 for 7 days (magnification x200).

After 7-day induction by SW620 supernatant, the expression of phospho-p44/42 ERK1/2 was still strong in the differentiating iDCs. In order to verify that the ERK1/2 signaling pathway is essential for the differentiation, the effect of PD98059 $10 \mu \mathrm{M}$ on the endothelial-like differentiation of iDCs was investigated. Results showed that ERK1/2 phosphorylation was obviously inhibited after 7-day induction by SW620 supernatant in the presence of PD98059 ( $n=3, p<0.01)$ (Fig. 6B). Inhibition of ERK1/2 phosphorylation by PD 98059 was accompanied by a significantly decreased expression of vWF and CD144 in induced cells $(n=3, p<0.01)$ (Fig. 6B). Furthermore, DiI-Ac-LDL uptake was reduced in the cells of iDCs induced group in the presence of PD98059 (Fig. 6C). Thus, inhibition of ERK1/2 phosphorylation by PD98059 results in the inhibition of endothelial-like differentiation of iDCs, both morphologically and functionally.

The nuclear translocation of phosphorylation of ERK1/2 is key to activate related transcription factors to lead to cell differentiation $(14,15)$. Before the induction by SW620 supernatant, the level of phospho-ERK1/2 (green) in iDCs was low (Fig. 6D). iDCs incubated with SW620 supernatant for 7 days showed the presence of phospho-ERK1/2 (green) in the nuclei (red) of differentiating iDCs (Fig. 6D). In the presence of PD98059 for 7 days, ERK1/2 phosphorylation as well as nuclear translocation were inhibited (Fig. 6D). Thus, the phosphorylation and translocation of ERK1/2 induced by SW620 supernatant is required for the endothelial-like differentiation of iDCs.

The blocking of VEGF-A in SW620 supernatant inhibits the activation of ERK1/2 and endothelial-like differentiation of iDCs. In this study, to determine the role of VEGF-A in the ERK1/2 signaling, we used VEGF-A Ab $(1 \mu \mathrm{g} / \mathrm{ml})$ to block VEGF-A in the SW620 supernatant to investigate its effect on ERK1/2 signaling. Western blotting showed that the level of phospho-ERK1/2 was drastically decreased in the VEGF-A blocking group comparing with the induced group $(n=3$, $\mathrm{p}<0.01$ ) (Fig. 7), which showed that VEGF-A played a starter role during the process of ERK1/2 activated by SW620 supernatant.

To determine the blocking effect of VEGF-A on the expression of EC surface markers, we further checked the expression of vWF and CD144 by Western blotting. The results showed that CD144 and vWF expression decreased in 

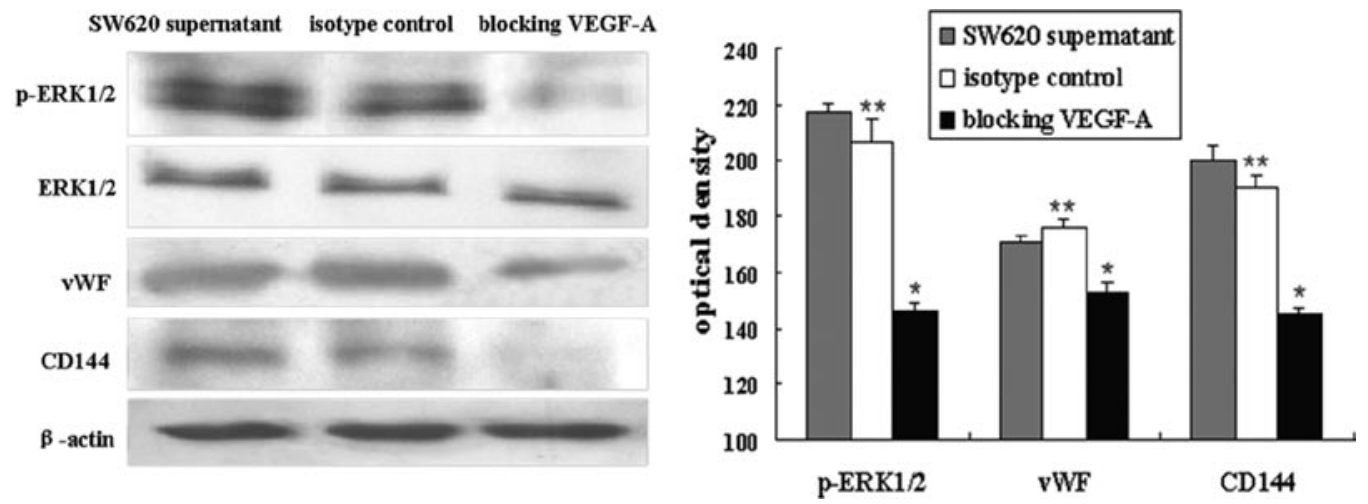

Figure 7. Western blot analysis of the expression of phospho-ERK1/2 and EC markers in cells blocking VEGF-A. The phospho-ERK1/2, vWF and CD144 were inhibited through blocking the VEGF-A in SW620 supernatant with VEGF-A Ab $1 \mu \mathrm{g} / \mathrm{ml}$. Results shown are the means \pm SD $(\mathrm{n}=3)$. ${ }^{*} \mathrm{p}<0.01 \mathrm{vs}$. SW620 supernatant group and isotype control; * ${ }^{*}>0.05$ vs. SW620 supernatant group.

the cells of the blocking VEGF-A group, compared with the induced cells $(n=3, p<0.01)$ (Fig. 7).

In brief, our results showed that VEGF-A not Ang2 mediates endothelial-like differentiation of iDCs by ERK1/2 signaling in the microenvironment of human colon adenocarcinoma.

\section{Discussion}

In this study, we first investigated the effect of the microenvironment produced by human colon adenocarcinoma cell line SW620 culture supernatant on the endothelial-like differentiation of iDCs derived from PBMCs. IDCs up-regulated the expression of the EC markers, while decreasing the DC markers in the presence of the microenvironment. Additionally, WP bodies and tube-like structures were formed, and DiI-Ac-LDL uptake was enhanced in these induced cells. All the above evidence showed that iDCs had differentiated into ELCs in this microenvironment. In fact, PBMCs act as pluripotent stem cells, which can be induced to acquire macrophage, endothelial, epithelial, and hepatocyte phenotypes (16). In this study, iDCs maintained some property of PBMCs, which led to differentiation toward ECs in specific microenvironment.

Tumor cell culture supernatant is often used in research in vitro. Addition of conditioned medium of murine Lewis lung carcinoma cells was used to investigate the $\mathrm{CD} 34^{+}$cells' differentiation (5). It has been shown that the tumor microenvironment characterized by the presence of cytokines and lactate can induce monocytes to transdifferentiate into ELCs in the presence of pro-angiogenic mediators $(6,9)$. These methods can simulate the tumor microenvironment to some extent. However, they are still far from the real tumor microenvironment in vivo. In fact, tumor development relies on a continuous cross-talk between cancer cells and their cellular and extracellular microenvironments, which includes complex and mutual interactions between tumor cells and various stromal cells $(17,18)$. To a certain extent, tumor tissue homogenate can better reflect the tumor microenvironment than the above methods. Previous researches has not proved what is the effect of normal tissue microenvironment on endothelial-like differentiation of iDCs. Therefore, next we used the homogenate supernatant of human colon adenocarcinoma and peri-carcinoma to simulate the tumor microenvironment, to investigate their effect on endothelial-like differentiation of iDCs. Due to the limitation of tumor specimens, we checked the effect of homogenate supernatant on cell morphology, the expression of $\mathrm{vWF}$ and CD144, and the function of DiI-Ac-LDL and India ink uptake. Results showed that similar to SW620 culture supernatant, homogenate supernatant of colon adenocarcinoma could induce endothelial-like differentiation of iDCs. However, peri-carcinoma supernatant had no obvious effect on it.

Various extracellular factors are involved in the process of cell differentiation. Among them, growth factors play a central role in the EC differentiation (19). Colon carcinomas produce both Ang 2 and VEGF, this coproduction likely initiates tumor blood vessel formation in a synergistic fashion (20). In a VEGF-rich milieu, destabilization of blood vessels by Ang 2 is followed by sprouting angiogenesis, whereas, in a VEGF-depleted state, Ang2 causes endothelial death and vessel regression $(21,22)$. Thus, the role of Ang2 in endotheliallike differentiation of iDCs deserves checking. In this study, results showed that blocking Ang2 in SW620 supernatant could not inhibit the endothelial-like differentiation of iDCs, which suggests Ang2 does not play a key role in this process of differentiation. It has been shown that $\mathrm{CD} 34^{+}$progenitor cells differentiation into ECs was blocked with sTie-2, suggesting that murine Lewis lung carcinoma-derived angiopoietins skewed the differentiation. However, the role of ang1 versus ang2 in this process is under study (5). Actually, Ang2 plays different roles in different tumor types. Lung and mammary carcinomas that overexpressed Ang2 and specific induction of Ang2 in gliomas were found to retard tumor growth and metastasis $(23,24)$.

VEGF has been shown to be an important cytokine that induces a series of biological effects in ECs $(25,26)$. Our previous study proved that blocking VEGF-A in EC9706 supernatant inhibited the endothelial-like differentiation of iDCs (8), which is consistent with the report that in mouse ovarian carcinomas, SU5416, a VEGF receptor tyrosine kinase inhibitor, abolished the vascular migration of $\mathrm{CD} 11 \mathrm{c}^{+}$cells, confirming the importance of tyrosine kinase signaling in DC endothelialization (1). In contrast, murine Lewis lung carcinoma-derived VEGF contributed to the increase in 
cellularity, but not to redirection of $\mathrm{CD} 34^{+}$differentiation into ECs (5). Therefore, it is worthwhile to check the role of VEGF-A in SW620 supernatant on the endothelial-like differentiation of iDCs.

Many growth factors can lead to cell differentiation through cellular signal transduction (27). The ERK1/2 signaling plays a key role in mediating signals from membrane receptors to the nucleus, and is involved in the processes of cell growth, differentiation, proliferation and apoptosis $(28,29)$. A study has shown that the ERK pathway plays a central role in VEGF-induced bone marrow stem cell differentiation into ECs (15). In this study, we observed that a sustained activation of the ERK1/2 is required for endothelial-like differentiation of iDCs. It is established that the nuclear translocation of phosphorylated ERK is key to the differentiation of various cells $(14,15,30)$. Our results demonstrated that activated ERK1/2 was present in the nucleus of cells incubated with SW620 supernatant. PD98059 not only inhibited the activation of ERK1/2, but also inhibited the nuclear translocation of activated ERK1/2.

Altogether, this study showed that iDCs could differentiate into ELCs in a colon adenocarcinoma microenvironment, and VEGF-A not Ang2 mediates endothelial-like differentiation of iDCs by ERK1/2 signaling in this microenvironment. There are likely two effects by which the tumor microenvironment could induce the differentiation of iDCs into ELCs. One is that vasculogenesis could further facilitate vascularization, which is required for tumor growth to progress $(5,7)$. The other possible effect is that the redirection of iDC differentiation might reduce the number of functional DCs, limiting the potential antitumor immune effect. In fact, many studies have shown that tumor patients have defects in DC maturation, and the number of functional DCs is reduced, which leads to immune suppression $(31,32)$.

Our study demonstrated a mechanism that could explain one of the reasons for the reduced number of tumor-infiltrating DCs. However, it is definitely worthwhile to further investigate the inhibition of the endothelial-like differentiation of DCs and enhancing their function in anti-tumor immunity.

\section{Acknowledgements}

We would like to express our thanks to Dr Fred Bogott, Dr Ziyou Cui and peer reviewers for critically reading this manuscript and giving good suggestions, and thanks to the open laboratory of the key discipline of molecular medicine of the Henan Province and the electron microscopy research center of the Hebei Medical University for their helpful technical support. This work was supported by the Science and Technology Research Key Project of Ministry of Education (No.207150) and the Natural Science Foundation of the Henan province of China (No. 20060011 and 2008A310018).

\section{References}

1. Conejo-Garcia JR, Benencia F, Courreges MC, Kang E, Mohamed-Hadley A, Buckanovich RJ, Holtz DO, Jenkins A, $\mathrm{Na}$, Zhang L, Wagner DS, Katsaros D, Caroll R and Coukos G: Tumor-infiltrating dendritic cell precursors recruited by a betadefensin contribute to vasculogenesis under the influence of VEGF-A. Nat Med 10: 950-958, 2004.
2. Conejo-Garcia JR, Buckanovich RJ, Benencia F, Courreges MC, Rubin SC, Carroll RG and Coukos G: Vascular leukocytes contribute to tumor vascularization. Blood 105: 679-681, 2005.

3. Coukos G, Benencia F, Buckanovich RJ and Conejo-Garcia JR: The role of dendritic cell precursors in tumour vasculogenesis. Br J Cancer 92: 1182-1187, 2005.

4. Coukos G, Conejo-Garcia JR, Buckanovich R and Benencia F: Vascular leukocytes: a population with angiogenic and immunosuppressive properties highly represented in ovarian cancer. Adv Exp Med Biol 590: 185-193, 2007.

5. Young MRI and Cigal M: Tumor skewing of CD34+ cell differentiation from a dendritic cell pathway into endothelial cells. Cancer Immunol Immunother 55: 558-568, 2006.

6. Gottfried E, Kreutz M, Haffner S, Holler E, Iacobelli M, Andreesen R and Eissner G: Differentiation of human tumourassociated dendritic cells into endothelial-like cells: an alternative pathway of tumour angiogenesis. Scand J Immunol 65: 329-335, 2007.

7. Silvano S, Marco R, Elena R, Stefania M and Marco P: Dendritic cell-endothelial cell cross-talk in angiogenesis. Trends Immunol 28: 385-392, 2007.

8. Lu J, Zhao J, Liu K, Zhao J, Yang H, Huang Y, Qin Z, Bai R, Li P, Ma J, Yan W, Zhao M and Dong Z: MAPK/ERK1/2 signaling mediates endothelial-like differentiation of immature DCs in the microenvironment of esophageal squamous cell carcinoma. Cell Mol Life Sci 67: 2091-2106, 2010.

9. Fernandez Pujol B, Lucibello FC, Zuzarte M, Lütjens P, Müller R and Havemann K: Dendritic cells derived from peripheral monocytes express endothelial markers and in the presence of angiogenic growth factors differentiate into endothelial-like cells. Eur J Cell Biol 80: 99-110, 2001.

10. Jemal A, Siegel R, Ward E, Hao Y, Xu J and Thun MJ: Cancer statistics, 2009. CA Cancer J Clin 59: 225-249, 2009.

11. Müftüoğlu TM, Köksal N and Ozkutlu D: Evaluation of phagocytic function of macrophages in rats after partial splenectomy. J Am Coll Surg 191: 668-671, 2000.

12. Aranguren XL, Luttun A, Clavel C, Moreno C, Abizanda G, Barajas MA, Pelacho B, Uriz M, Arana M, Echavarri A, Soriano M, Andreu EJ, Merino J, Garcia-Verdugo JM, Verfaillie CM and Prosper F: In vitro and in vivo arterial differentiation of human multipotent adult progenitor cells. Blood 109: 2634-2642, 2007.

13. Li Z, Wu JC, Sheikh AY, Kraft D, Cao F, Xie X, Patel M, Gambhir SS, Robbins RC, Cooke JP and Wu JC: Differentiation, survival, and function of embryonic stem cell derived endothelial cells for ischemic heart disease. Circulation 116: 46-54, 2007.

14. Ramos JW: The regulation of extracellular signal-regulated kinase (ERK) in mammalian cells. Int J Biochem Cell Biol 40: 2707-2719, 2008 .

15. Xu J, Liu X, Jiang Y, Chu L, Hao H, Liua Z, Verfaillie C, Zweier J, Gupta K and Liu Z: MAPK/ERK signalling mediates VEGFinduced bone marrow stem cell differentiation into endothelial cell. J Cell Mol Med 12: 2395-2406, 2008.

16. Zhao Y, Glesne D and Huberman E: A human peripheral blood monocyte-derived subset acts as pluripotent stem cells. Proc Natl Acad Sci USA 100: 2426-2431, 2003.

17. Gout $\mathrm{S}$ and Huot $\mathrm{J}$ : Role of cancer microenvironment in metastasis: focus on colon cancer. Cancer Microenvironment 1: 69-83, 2008.

18. Ribatti D and Vacca A: The role of microenvironment in tumor angiogenesis. Genes Nutr 3: 29-34, 2008.

19. Stavridis MP, Lunn JS, Collins BJ and Storey KG: A discrete period of FGF-induced Erk1/2 signalling is required for vertebrate neural specification. Development 134: 2889-2894, 2007.

20. Ahmad SA, Liu W, Jung YD, Fan F, Reinmuth N, Bucana CD and Ellis LM: Differential expression of angiopoietin-1 and angiopoietin-2 in colon carcinoma. A possible mechanism for the initiation of angiogenesis. Cancer 92: 1138-1143, 2001.

21. Augustin HG, Koh GY, Thurston G and Alitalo K: Control of vascular morphogenesis and homeostasis through the angiopoietin-Tie system. Nat Rev Mol Cell Biol 10: 165-177, 2009.

22. Jin SW and Patterson C: The opening act: vasculogenesis and the origins of circulation. Arterioscler Thromb Vasc Biol 29: 623-629, 2009.

23. Machein MR, Knedla A, Knoth R, Wagner S, Neuschl E and Plate KH: Angiopoietin-1 promotes tumor angiogenesis in a rat glioma model. Am J Pathol 165: 1557-1570, 2004.

24. Yu Q and Stamenkovic I: Angiopoietin-2 is implicated in the regulation of tumor angiogenesis. Am J Pathol 158: 563-570, 2001. 
25. Lee HT, Chang YC, Tu YF and Huang CC: VEGF-A/VEGFR-2 signaling leading to cAMP response element-binding protein phosphorylation is a shared pathway underlying the protective effect of preconditioning on neurons and endothelial cells. J Neurosci 29: 4356-4368, 2009.

26. Huh JE, Baek YH, Lee MH, Choi DY, Park DS and Lee JD: Bee venom inhibits tumor angiogenesis and metastasis by inhibiting tyrosine phosphorylation of VEGFR-2 in LLC-tumor-bearing mice. Cancer Lett 292: 98-110, 2010.

27. Craig EA, Austin AF, Vaillancourt RR, Barnett JV and Camenisch TD: TGF 32 -mediated production of hyaluronan is important for the induction of epicardial cell differentiation and invasion. Exp Cell Res 316: 3397-3405, 2010.

28. Anfuso CD, Giurdanella G, Motta C, Muriana S, Lupo G, Ragusa N and Alberghina M: PKCalpha-MAPK/ERK-phospholipase A2 signaling is required for human melanoma-enhanced brain endothelial cell proliferation and motility. Microvasc Res 78: 338-357, 2009 .
29. Poli M, Luscieti S, Gandini V, Maccarinelli F, Finazzi D, Silvestri L, Roetto A and Arosio P: Transferrin receptor 2 and HFE regulate furin expression via mitogen-activated protein kinase/extracellular signal-regulated kinase (MAPK/Erk) signaling. Implications for transferrin-dependent hepcidin regulation. Haematologica 95: 1832-1840, 2010.

30. Crompton T, Gilmour KC and Owen MJ: The MAP kinase pathway controls differentiation from double-negative to doublepositive thymocyte. Cell 86: 243-251, 1996.

31. Muthana M, Fairburn B, Mirza S, Slack LK, Hopkinson K and Pockley AG: Identification of a rat bone marrow-derived dendritic cell population which secretes both IL-10 and IL-12: evidence against a reciprocal relationship between IL-10 and IL-12 secretion. Immunobiology 211: 391-402, 2006.

32. Frenzel H, Pries R, Brocks CP, Jabs WJ, Wittkopf $\mathrm{N}$ and Wollenberg B: Decreased migration of myeloid dendritic cells through increased levels of C-reactive protein. Anticancer Res 27: 4111-4115, 2007. 